had normal neurologic and developmental outcomes. In contrast, of 10 with hydrocephalus ex vacuo from birth, eight had severe disabilities. Nine of 34 (26\%) children had microcephaly. Of those presenting with chorioretinal lesions (69\%), the majority had residual visual loss after therapy. Risk factors for poor outcome included diabetes insipidus, hypoxia, hydrocephalus with high CSF protein, and delay in medical treatment. These results compared to previous reports for untreated children were thought to justify treatment of pregnant women with acute gestational Toxoplasma infection and young infants with congenital toxoplasmosis. (Roizen N, Swisher CN et al. Neurologic and developmental outcome in treated congenital toxoplasmosis. Pediatrics January 1995;95:11-20). (Reprints:Dr Rima McLeod, 114 Baumgarten, Department of Medicine, Michael Reese Hospital, 2929 South Ellis Ave, Chicago, IL 60616).

COMMENT. One third of the treated patients were severely impaired neurologically, and two thirds of those with normal developmental outcomes had retinal lesions and visual problems. The need for prevention and improved therapies was emphasized.

\title{
CT AND IQ IN HIV DISEASE
}

Measures of cognitive function and social-emotional behavior were correlated with CT abnormalities in 87 children with symptomatic human immunodeficiency virus type 1 disease (HIV) at the Pediatric Branch, National Cancer Institute, and the NIND \& S, Bethesda, MD; Children's National Medical Center, Washington, DC; and Medical Illness Counselling Center, Chevy Chase, MD. The mean age of the patients was 4.3 yrs. Vertically infected children were $2.3+/-0.3$ years, and transfusion-infected children were $8.4+/-0.6$ years of age. The Full Scale IQ (FIQ) was a mean of 85.2 for the total group; 80 for vertically infected; and 95.5 for transfusion-infected patients. A significant correlation was found between FIQ and the overall CT severity rating. The correlation was stronger in (younger) vertically infected compared with older transfusion-infected children. Calcifications, observed only in vertically infected children (16 of 58), were associated with greater delays in neurocognitive development, independent of the degree of brain atrophy. (Brouwers P, DeCarli C et al. Correlation between computed tomographic brain scan abnormalities and neuropsychological function in children with symptomatic human immunodeficiency virus disease. Arch Neurol Jan 1995;52:39-44). (Reprints: Dr Brouwers, Pediatric Branch, National Cancer Institute, National Institutes of Health Clinical Center, Room 13N240, Bethesda, MD 20892).

COMMENT. CT scans are recommended as a baseline for patients at risk for CNS manifestations and cognitive deficits due to HIV. Even when mild, CT abnormalities were of clinical significance.

The above authors have studied the effects of HIV disease on receptive and expressive language in 36 children with symptomatic HIV and the relation to CT scan brain abnormalities (Wolters PL, Brouwers P et al. Pediatrics Jan 1995;95:112-119). Expressive language was more impaired than receptive language. Greater severity of CT abnormalities was correlated with poorer receptive and expressive language functioning. The language impairments were associated with the direct effects of HIV-related CNS disease.

A speech motor control disorder developed after HIV infection in 6 right-handed patients. They had an ataxic dysarthria, associated with ataxic gait and intention tremors. The motor speech disorder was due to a cerebellar dysfunction. (Lopez OL et al. Neurology 1994;44:2187-2189). 